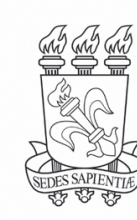

\title{
Artigos
}

\section{Avaliação da perda da biodiversidade na Mata Atlântica}

\author{
Assessment of biodiversity loss in the Atlantic Forest
}

\author{
Antonia Francivan Vieira Castelo Branco ${ }^{\circ}$ \\ Patrícia Verônica Pinheiro Sales Lima' ${ }^{\bullet}$ \\ Esdras Soares de Medeiros Filho' ${ }^{\bullet}$ \\ Benedita Marta Gomes Costa" ${ }^{\odot}$ \\ Tarcisio Praciano Pereirall ${ }^{\odot}$
}

'Universidade Federal do Ceará, Fortaleza, CE, Brasil

"Universidade Estadual Vale do Acaraú, Sobral, CE, Brasil

\section{RESUMO}

A biodiversidade da Mata Atlântica é considerada de extrema importância biológica, mas essa riqueza de fauna e flora está ameaçada pelo desmatamento. O objetivo deste estudo consiste em apresentar um modelo de avaliação da perda da biodiversidade, adotando como cenário o município de Viçosa do Ceará, situado no Nordeste brasileiro. O método adotado baseia-se na proposição de um indicador em que se busca calcular a estimativa da perda da biodiversidade a partir do decréscimo da riqueza de aves, mamíferos e répteis escamados que fazem parte desse habitat, tendo como referência o modelo da relação espécie-área. Como resultados tem-se: (1) a perda da biodiversidade na área de estudo está em nível "extremo"; (2) as taxas de variação média e de velocidade têm movimento acelerado, logo, quanto maior for o aumento da área desmatada, mais rápida se torna a velocidade e aceleração da perda da biodiversidade; (3) a curvatura da função indicou que o ponto crítico da perda da biodiversidade acontece quando o desmatamento atinge $93 \%$ da área do ecossistema. Conclui-se que o modelo de estimativa da perda de aves, mamíferos e répteis escamados apresentou-se de forma eficiente para a avaliação da perda da biodiversidade no ecossistema de Mata Atlântica.

Palavras-chave: Relação espécie-área; Modelo matemático; Estimativa; Coeficiente 


\begin{abstract}
The Atlantic Forest biodiversity is considered of extreme biological importance; however, its fauna and flora richness has been threatened by deforestation. This study aimed to present a model for assessing biodiversity loss, considering the municipality of Viçosa do Ceará (northeastern Brazil) as the study area. The used method is based on the proposal of an indicator, from which, we sought to calculate the estimate of biodiversity loss through the decrease in the richness of birds, mammals, and scaly reptiles in the studied habitat, using the species-area relationship model as a reference. The following results were obtained: (1) the biodiversity loss in the study area is at an "extreme" level; (2) the rates of average variation and speed have accelerated movement, therefore, the greater the increase of deforested area, the faster the speed and acceleration of biodiversity loss; (3) the curvature of the function indicated that the critical point of biodiversity loss is reached when deforestation reaches $93 \%$ of the ecosystem area. It was concluded that the model used to estimate the loss of birds, mammals, and scaly reptiles was efficient to assess biodiversity loss in the Atlantic Forest ecosystem.
\end{abstract}

Keywords: Species-area relationship; Mathematical model; Estimate; Coefficient

\title{
1 INTRODUÇÃo
}

Durante a realização da Conferência das Nações Unidas sobre o Meio Ambiente e o Desenvolvimento, também denominada Eco-92, foi declarado que as ações humanas estavam eliminando genes, espécies e características biológicas em ritmo acelerado. Para Cardinale et al. (2012), as discussões buscando compreender e mensurar a perda da biodiversidade e suas consequências sobre o ecossistema têm sido latentes no meio científico.

Circunscritos nessa temática têm surgido pesquisas que buscam analisar a perda da biodiversidade adotando modelos matemáticos a fim de estimar os impactos das ações humanas sobre o meio ambiente. Dentre os estudos anteriores realizados sobre esta matéria destacam-se as análises de Chaudhary e Mooers (2018) e Chisholm et al. (2018), que buscam analisar e/ou estruturar um modelo para verificar os impactos ocasionados pela perda da biodiversidade na sustentabilidade dos ecossistemas.

De forma geral, Chisholm et al. (2018) demonstram que não existe um indicador universal que reflita com precisão as mudanças na biodiversidade considerando os diferentes ecossistemas e escalas espaciais, por isso a necessidade de se apresentar 
modelos envolvendo cenários específicos. No espectro desses estudos e visando contribuir com essa temática, o presente trabalho tem como objetivo geral apresentar um modelo de avaliação da perda da biodiversidade, adotando como cenário o município de Viçosa do Ceará, situado no Nordeste brasileiro. Tem-se como objetivos específicos: estimar a velocidade e aceleração da perda da biodiversidade a partir do modelo gerado; encontrar o ponto crítico da perda da biodiversidade; propor um coeficiente de perda da biodiversidade.

O modelo de avaliação da perda da biodiversidade deste estudo consiste na estimativa de perda referente às classes de mamíferos, aves e répteis escamados. Para tanto, foi levado em consideração que a perda da biodiversidade consiste em um problema global, mas a sua ocorrência é um fenômeno localizado (JURAS, 2010). Nessa perspectiva, foram adotados como cenário de aplicação e verificação do modelo os dados referentes ao remanescente de Mata Atlântica localizado no município de Viçosa do Ceará, situado na região Nordeste do Brasil. O referido espaço é o detentor do maior enclave desse bioma no Estado do Ceará. No entanto, a exploração desordenada dos recursos naturais provocou uma redução de 75\% na vegetação original do enclave de Mata Atlântica no Município.

\section{REFERENCIAL TEÓRICO}

\subsection{Perda da biodiversidade}

De acordo com Veiga e Ehlers (2003), o termo "biodiversidade" foi criado na década de 1980, pelo biólogo norte-americano Edward O. Wilson. A biodiversidade (ou diversidade biológica) se refere a: diversidade de espécies - variedade de espécies existentes em uma área; diversidade genética - refere-se a diferentes populações dentro da mesma espécie; diversidade ecológica - cada ecossistema abriga uma combinação característica de plantas e animais. Neste trabalho, a abordagem sobre biodiversidade estará relacionada à diversidade de espécies. 
Na opinião de Veiga e Ehlers (2003), os fenômenos que mais provocam a perda da biodiversidade são: destruição e alteração de habitats, exploração de espécies "selvagens", introdução de espécies exóticas, homogeneidade, poluição e mudanças ambientais globais. Para Haddad et al. (2015), a maior ameaça à biodiversidade é a perda de habitats. De acordo com Santos (2003), estudos voltados para analisar a perda de biodiversidade possibilitam propor estratégias de monitoramento rápido da diversidade biológica, assim como o desenvolvimento de programas de conservação e o uso sustentável de recursos biológicos, que são as formas conhecidas para desacelerar a perda da biodiversidade.

Uma forma de preservar a maioria das espécies com um menor custo é através da identificação de hotspots de biodiversidade, sendo áreas com excepcional concentração de espécies endêmicas que estão perdendo seu habitat. Os hotspots são locais do planeta que concentram espécies endêmicas ameaçadas (MYERS et al., 2000; HOPPER; SILVEIRA; FIEDLER, 2016) e área inferior a 30\% da cobertura vegetal original (MYERS et al., 2000).

A Floresta Atlântica é um dos 36 hotspots globais de biodiversidade (REZENDE et al., 2018). A conservação dos hotspots implica a diminuição da perda da biodiversidade observada nos mais diversos ecossistemas terrestres.

\subsection{Degradação e fragmentação da Mata Atlântica}

As florestas tropicais estão sendo fragmentadas em todo o mundo (ARROYORODRIGUEZ et al., 2017). Essa fragmentação florestal provoca perda da biodiversidade (FERREIRA et al., 2019). A Mata Atlântica é uma das florestas tropicais mais ameaçadas do planeta (SAFAR; MAGNAGO; SCHAEFER, 2020). Atualmente, esse bioma vem sendo devastado com o processo de urbanização (FERREIRA et al., 2019), pois mais de 60\% da população brasileira reside em área de domínio de Mata Atlântica (SCARANO; CEOTTO, 2015). 
Em meio à área de domínio paisagístico da Caatinga do Nordeste há enclaves de mata úmida evidenciando um contraste no ambiente. Os remanescentes de Mata Atlântica são uma paisagem de exceção no Nordeste, podendo-se comparar "ilhas" de mata úmida no "mar" de Caatinga. Os enclaves de florestas úmidas são lugares de maior riqueza de vida selvagem do Nordeste (CAVALCANTE, 2005).

Entre os grupos de animais mais vulneráveis à extinção, devido à fragmentação de ecossistemas, destacam-se os grandes carnívoros, as aves que habitam o interior da floresta e os vertebrados de médio e grande portes que se alimentam de frutas. A maior parte das espécies brasileiras ameaçadas de extinção ocorre na Mata Atlântica. As maiores ameaças à diversidade biológica são a destruição e a fragmentação dos ambientes naturais (TABARELLI; PINTO; LEAL, 2009; HADDAD et al., 2015).

\section{MATERIAL E MÉTODOS}

A proposição do modelo de avaliação da perda da biodiversidade deste estudo consiste na estimativa de perda referente às classes de mamíferos, aves e répteis escamados. O modelo desenvolvido encontra o nível de perda de biodiversidade a partir do cálculo do coeficiente, cujo resultado classifica o estado dessa perda em relação ao ambiente preservado como sendo de extremo, alto, médio, baixo ou insignificante. As etapas do modelo são apresentadas através da Figura 1.

Com base na Figura 1 é possível verificar que a aplicação do modelo adota os seguintes passos: toma-se o modelo da relação espécie-área adequando-o para o estudo da perda da biodiversidade (considerando a perda de espécies em função da área desmatada) das classes de aves, mamíferos e répteis escamados; a partir do modelo da perda faz-se a estimativa de espécies para o cenário ambiente preservado e, em seguida, verifica-se a perda a partir desse estágio inicial de análise (1985); com base nesse resultado verifica-se a velocidade (taxa de variação média), a aceleração 
(taxa de variação da velocidade) e o ponto crítico de perda; toma-se o modelo de extinção local a partir do estágio inicial de análise (1985) e fazendo comparação com a perda ocorrida até o ano de 2014 encontra-se o coeficiente da perda da biodiversidade, que revela o nível de perda de riqueza de espécies.

Figura 1 - Fluxograma do modelo de avaliação da perda da biodiversidade

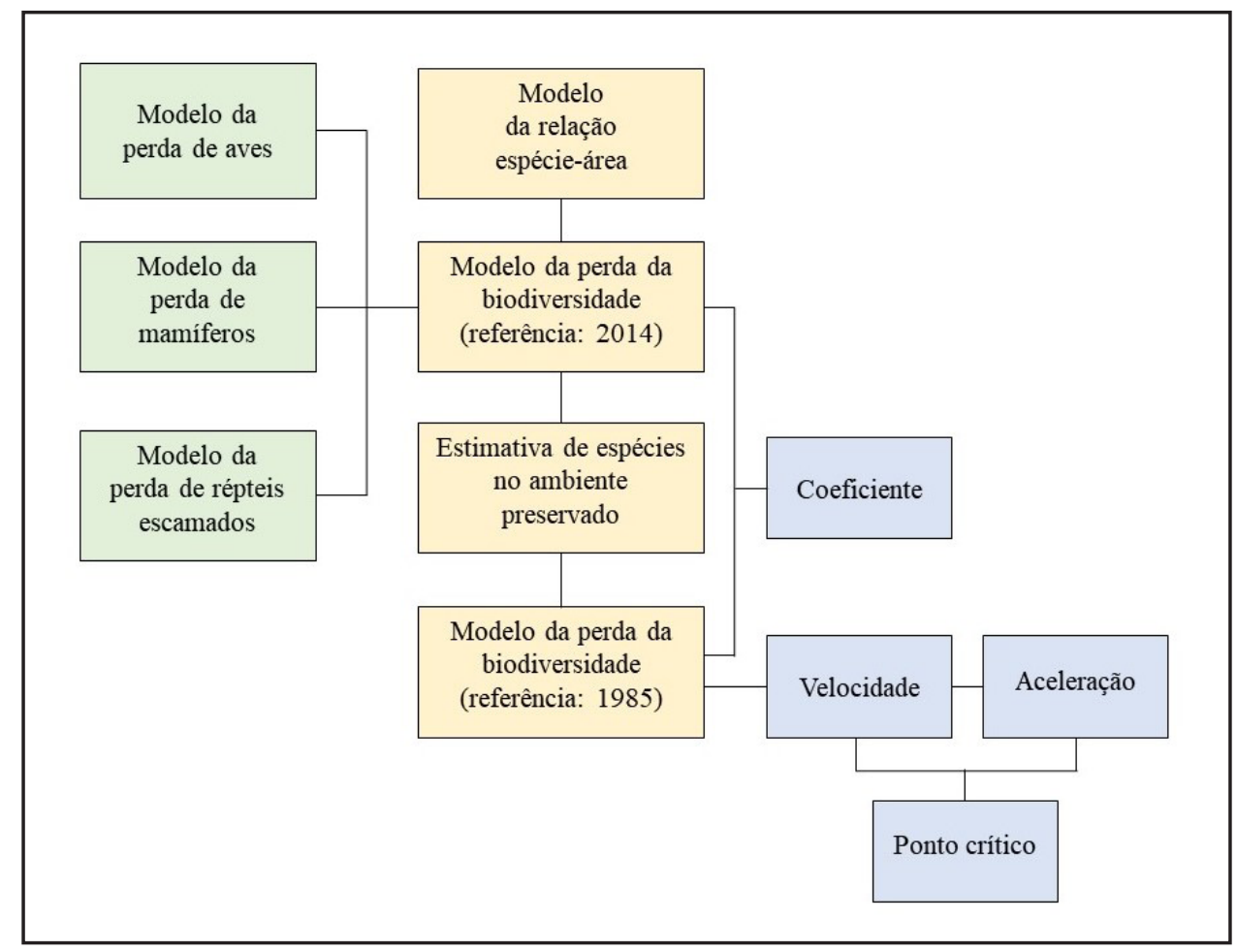

Fonte: Autores (2020)

Para aplicação do modelo proposto foram obtidos dados de estudos anteriores referentes à extinção local de animais vertebrados, das classes de mamíferos, aves e répteis escamados, no município de Viçosa do Ceará, localizado na região Nordeste do Brasil. As informações foram obtidas através da comparação das relações de espécies locais, no espaço temporal, apresentadas por Barros (1980), Ceará (1998) e Ibama (1998).

O item a seguir busca descrever as equações adotadas na construção do modelo da perda da biodiversidade. 


\subsection{Modelo da relação espécie-área}

O modelo da relação espécie-área, ou riqueza de espécies, é apresentado por MacArthur e Wilson (2001), conforme expresso na Equação (1):

$$
s=c \cdot a^{2}
$$

Em que: $s$ = quantidade de espécies (ou riqueza); $c$ = constante referente ao ecossistema; $a=$ área do habitat; $z$ = parâmetro de riqueza de espécies. A Equação (1) tem s como uma variável dependente de $a$. Segundo Purves et al. (2005), a riqueza de espécies constitui a medida mais simples para mensurar a diversidade. Santos et al. (2009) são um exemplo de estudo realizado envolvendo a teoria da relação espécie-área.

Partindo da Equação (1) e inserindo a variável área desmatada, pode-se obter uma equação para representar a perda de riqueza de espécies em função do decréscimo da cobertura vegetal, resultando na Equação (2):

$$
s(x)=c
$$

Em que: $x=$ área desmatada. Vê-se que a extensão devastada $(\mathrm{x})$ cresce até o limite $a$. Então o valor de $\mathrm{s}(\mathrm{x})$ tende a zero quando $\mathrm{x}$ se aproxima de $\mathrm{a}$, que é o máximo possível de desmatamento da área observada e que resultaria na anulação dos animais.

Para a aplicação na Equação (2) foram adotadas como parâmetro as informações do município de Viçosa do Ceará, que possuía uma área original de Mata Atlântica equivalente a 39.075 ha, restando 9.610 ha de remanescente do ecossistema (FUNDAÇÃO SOS MATA ATLÂNTICA; INSTITUTO NACIONAL DE PESQUISAS ESPACIAIS, 2015). A Mata Atlântica do município abriga 55 espécies de aves, 24 espécies de mamíferos terrestres de pequeno, médio e grande portes, e 18 espécies de répteis escamados (IBAMA, 1998). De acordo com Santos (2003), devido à diversidade de seres que podem ser encontrados em qualquer ambiente, é praticamente impossível determinar a sua riqueza biológica de forma precisa.

Assim, tomando-se a Equação (2), considera-se $z=0,203$, sendo o valor normalmente utilizado para vertebrados terrestres (MACHADO et al., 2008), e 
aplicando: o número de espécies de aves equivalente a 55, a quantidade de espécies de mamíferos igual a 24 e a quantidade de espécies de répteis igual a 18; a área original do ecossistema (a) correspondente a 39.075 ha; e a área desmatada (x) equivalente a 29.465 ha, calculou-se a constante c para os grupos de vertebrados terrestres. Assim, a estimativa de perda de espécies de aves $(v(x))$, mamíferos $(m(x))$ e répteis $(r(x))$, pode ser expressa através das Equações (3), (4) e (5), respectivamente.

$$
\begin{aligned}
& v(x)=8,548 \cdot(9.610-x)^{0,203} \\
& m(x)=3,73 \cdot(9.610-x)^{0,203} \\
& r(x)=2,798 \cdot(9.610-x)^{0,203}
\end{aligned}
$$

A Equação da perda da biodiversidade (6) foi gerada a partir da somatória das Equações (3), (4) e (5):

$$
s(x)=15,076 \cdot(9.610-x)^{0,203}
$$

\subsection{Estimativa de espécies nos cenários de ambiente preservado e degradado}

Tomando-se a Equação (6) e transformando-a na relação espécie-área, encontrou-se a riqueza de espécies s1( $x$ ) em função da área do ecossistema $(x)$ :

$$
s 1(x)=15,076 \cdot x^{0,203}
$$

Quando a cobertura de Mata Atlântica abrangia 39.075 ha, que representa a totalidade da área original do ecossistema no Município, então s1(39.075) apontava o valor de aproximadamente 129 espécies da fauna existentes no cenário de ambiente preservado.

Tomando-se a Equação (7) em que: $x=$ a área original do ecossistema (39.075 
ha), s1(x) = quantidade de espécies da fauna (129) e z = constante $(0,203)$, o resultado foi que a constante $c$ da equação, que estimou a perda da biodiversidade a partir do cenário de ambiente preservado, teve valor equivalente a 15,081. Assim, a estimativa de perda de diversidade de espécies a partir desse cenário foi expressa pela Equação (8):

$$
\operatorname{so}(x)=15,081
$$

\subsection{Taxa de variação média e taxa de variação da velocidade da perda da biodiversidade}

A taxa de variação média é a velocidade da perda de riqueza de espécies. A derivada da Equação (2) gera a Equação (9), que representa a velocidade com que acontece a perda de riqueza de espécies. A taxa de variação média para o cenário de ambiente preservado foi encontrada através da derivação da função (8), resultando na Equação (10). As funções $d s(x)$ e $d s o(x)$ apresentam a relação espécie/ha (da área destruída).

$$
\begin{aligned}
& d s(x)=-c \cdot z \cdot(a-x)^{z-1} \\
& d s o(x)=-3,061 .(39.075-x)^{-0,797}
\end{aligned}
$$

A derivada de segunda ordem da função (2) gera a Equação (11), que representa a aceleração em que acontece perda de espécies. A taxa de variação da velocidade (aceleração), da perda a partir do cenário de ambiente preservado foi encontrada através da derivação de segunda ordem da função (8), que gera a Equação (12).

$$
\begin{aligned}
& d 2 s(x)=c \cdot z \cdot(z-1) \cdot(a-x)^{z-2} \\
& d 2 s o(x)=-2,44 \cdot(39.075-x)^{-1,797}
\end{aligned}
$$




\subsection{Curvatura e ponto crítico}

A equação da curva apontou o quanto ela se intensifica em diferentes pontos. O comportamento dessa inclinação é expresso pela Equação (13):

$$
k(x)=\frac{|d 2 s(x)|}{\left[1+(d s(s))^{2}\right]^{\frac{3}{2}}}
$$

O comportamento da curvatura do gráfico gerado pela função (8) foi verificado através da função (14), expressa por:

$$
k o(x)=\frac{2,44 \cdot(39.075-x)^{-1,797}}{\left[1+9,37 \cdot(39.075-x)^{-1,594}\right]^{\frac{3}{2}}}
$$

Generalizando o comportamento da curvatura do gráfico para quaisquer ecossistemas foi gerada a Equação (15), em função da área desmatada em percentual.

$$
k(x)=\frac{6,325 \cdot(100-x)^{-1,797}}{\left[1+63,521 \cdot(100-x)^{-1,594}\right]^{\frac{3}{2}}}
$$

A curvatura máxima é chamada de "beira do abismo". Entende-se por "beira do abismo" o ponto de maior curvatura do gráfico, o momento em que a curva se assemelha à beira de um penhasco, indicando que a partir desse ponto a perda acontece de forma crítica devido à rapidez da extinção local de espécies por área. Esse ponto foi encontrado através do zero da função (16), obtida através da derivação da Equação (13).

$$
d k(x)=\frac{3 c^{3} z^{3}(z-1)(2 z-2)(a-x)^{3 z-5}}{2\left[1+c^{2} z^{2}(a-x)^{2 z-2}\right]^{\frac{5}{2}}}-\frac{c z(z-1)(z-2)(a-x)^{z-3}}{\left[1+c^{2} z^{2}(a-x)^{2 z-2}\right]^{\frac{3}{2}}}
$$

A Equação (17) foi obtida por meio da derivação da função (15). O zero da função $d k o(x)$ define o ponto principal de inclinação da função (15), apontando a intensidade na curvatura do fenômeno de perda da biodiversidade. A Equação (17) se aplica a quaisquer ecossistemas, pois está em função do percentual de área desflorestada.

$$
d k(x)=\frac{11,416 \cdot(100-x)^{-2,797}}{\left[1+63,53(100-x)^{-1,594}\right]^{\frac{3}{2}}}-\frac{964,96 \cdot(100-x)^{-4,391}}{\left[1+63,53(100-x)^{-1,594}\right]^{\frac{5}{2}}}
$$


A determinação do ponto crítico ou "beira do abismo" é de grande relevância, pois indica o tamanho crítico da área do ecossistema a ser preservado. A partir desse ponto a queda das espécies será de maneira acentuada.

\subsection{Coeficiente da perda da biodiversidade}

A construção do coeficiente da perda da biodiversidade partiu da relação espécie-área e foi inspirada na Curva de Lorenz e no Coeficiente de Gini, que mensura no intervalo de 0 a 1 a desigualdade de distribuição de renda em relação à curva de perfeita distribuição de renda da população. O clímax é o nível de máxima diversidade biológica de um ecossistema natural, onde acontece o auge do equilíbrio entre as espécies. Para verificação do nível de perda da biodiversidade já ocorrida no ecossistema admite-se o cenário de ambiente preservado como referência. A Figura 2 apresenta a comparação entre o cenário de preservação $(P)$ e o cenário de perda de biodiversidade ocorrida pelo desmatamento (D), que aponta a redução de área de cobertura vegetal e a diminuição da quantidade de espécies de animais. Subtraindose da área preservada o espaço que foi desmatado, tem-se a remanescente (R).

Figura 2 - Diagrama conceitual de perda da biodiversidade

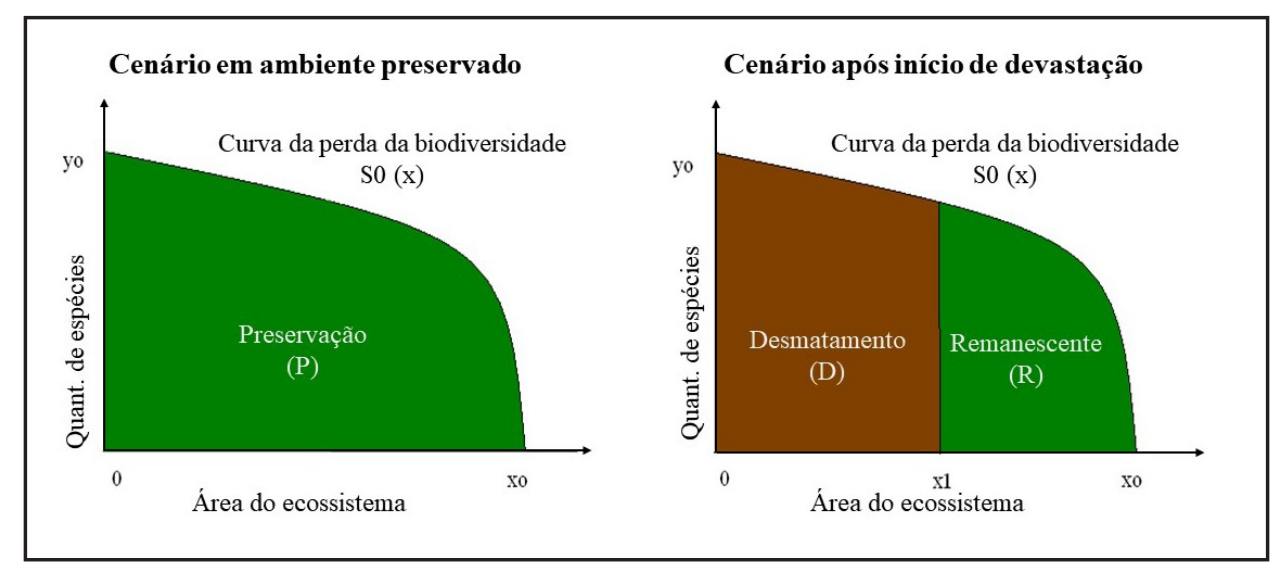

Fonte: Autores (2020)

Assim, o coeficiente da perda da biodiversidade $\left(C_{\mathrm{PB}}\right)$ é obtido através da razão entre a área do cenário do ecossistema com desmatamento (D) e a área do cenário 
original preservado $(P)$, sendo que $(P)$ é equivalente à soma da área desmatada (D) com a área remanescente $(R)$. A área $(P)$ do gráfico representa o estado nulo de perda da biodiversidade, pois parte de um cenário em que não houve redução da cobertura vegetal. Essa área é obtida através da integral definida de 0 (origem) a x0 (área total do ecossistema) da função s0(x), que representa o comportamento da curva da perda da biodiversidade, caso haja desmatamento. A área (D) do gráfico representa a perda da biodiversidade a partir da ocorrência de desmatamento, sendo obtida pela integral definida de 0 (origem) a x1 (tamanho da área desmatada no ecossistema) da função s0(x). Assim, tem-se o coeficiente de perda da biodiversidade expresso da forma:

$$
C_{P B}=\frac{\int_{0}^{x 1} \operatorname{so}(x) d(x)}{\int_{0}^{x 0} \operatorname{so}(x) d(x)}
$$

Em que: $0 \leq C_{P B} \leq 1$, sendo que se considera $C_{P B}=0$ quando o ecossistema se encontrar no cenário de ambiente preservado, ou seja, com ausência de perda da biodiversidade; e $C_{P B}=1$ quando o ecossistema estiver totalmente devastado, anulando toda a cobertura vegetal, assim como as espécies da fauna.

Tomando como referência esse intervalo na Figura 3 e Tabela 1, é possível apresentar uma proposta de classificação do nível de perda da biodiversidade a partir do resultado do coeficiente calculado na Equação (18).

Figura 3 - Diagrama conceitual do nível de perda da biodiversidade conforme o respectivo coeficiente

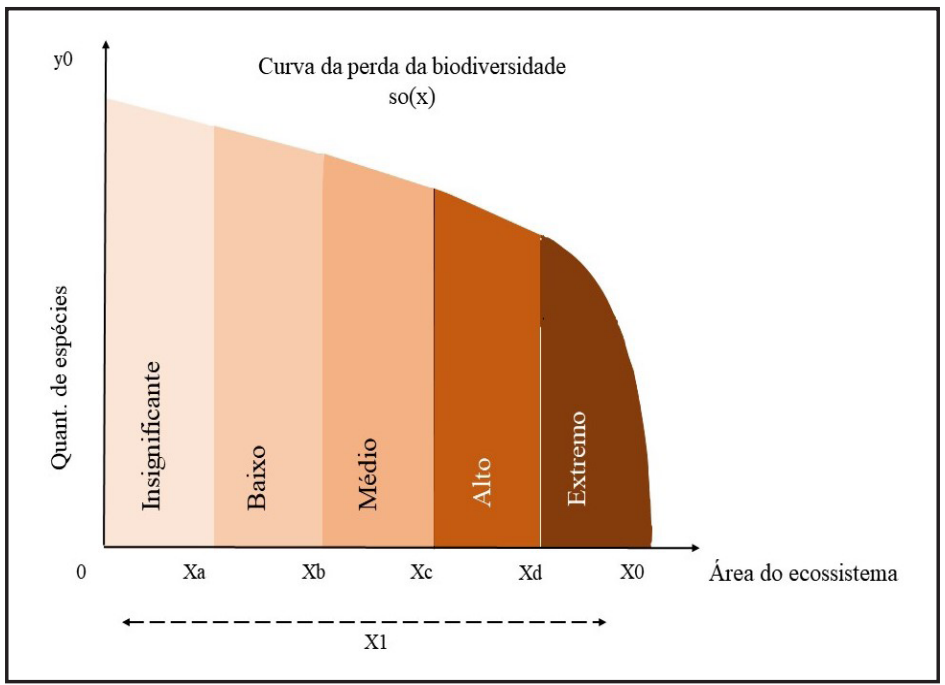

Fonte: Autores (2020) 
A Figura 3 mostra que à medida que o desmatamento avança na área do ecossistema, decresce a quantidade de espécies da fauna e intensifica a perda da biodiversidade. Para classificação do nível de perda da biodiversidade adotou-se quantidade ímpar de categorias para se estabelecer um intervalo mediano. Assim, foram definidas cinco categorias de nível de perda da biodiversidade e estabelecido o intervalo do valor do coeficiente de 0,4 a 0,6 como intermediário, sendo padronizada a amplitude de 0,2 em todas as classes dos intervalos. Para valores do coeficiente abaixo de 0,4 tem-se níveis inferiores de perda da biodiversidade, e para valores acima de 0,6 tem-se níveis mais elevados de perda da biodiversidade. Quando o valor do coeficiente for superior a 0,8 associa-se ao nível máximo de perda da cobertura vegetal e extinção de espécies da fauna, sendo que tende a ocorrer o ponto crítico da perda da biodiversidade, conforme apresentado nas Equações (16) e (17). Essa classificação é importante para se ter uma referência de quão grave está o nível de perda da biodiversidade desse ecossistema.

Tabela 1 - Classificação do nível de perda da biodiversidade conforme o respectivo coeficiente

\begin{tabular}{cc}
\hline Resultado do coeficiente & Nível de perda da biodiversidade \\
\hline $0 \leq \mathrm{CPB}<0,2$ & Muito baixo \\
$0,2 \leq \mathrm{CPB}<0,4$ & Baixo \\
$0,4 \leq \mathrm{CPB}<0,6$ & Médio \\
$0,6 \leq \mathrm{CPB}<0,8$ & Alto \\
$0,8 \leq \mathrm{CPB} \leq 1$ & Extremo \\
\hline
\end{tabular}

Fonte: Autores (2020)

Em que: $C_{P B}=$ coeficiente de perda da biodiversidade.

Neste estudo, a construção do coeficiente a partir da relação entre áreas avalia o nível de perda da biodiversidade considerando o agrupamento das variáveis: área desmatada e perda de espécies de animais. A comparação entre as áreas geradas referentes ao cenário com dados mais antigos da cobertura vegetal preservada e o cenário atual, com ocorrência de desmatamento, permite uma melhor compreensão do nível de devastação do ecossistema natural. 
Na construção de um coeficiente para representar a perda de espécies adotaram-se os cenários comparativos: 1985 como o ano mais longínquo com dados da cobertura vegetal preservada, e 2014 como o cenário mais recente sobre a extensão do bioma no município (FUNDAÇÃO SOS MATA ATLÂNTICA; INSTITUTO NACIONAL DE PESQUISAS ESPACIAIS, 2009; 2015). Assim, tomando-se o primeiro cenário em que se encontrava um melhor estado de conservação do ecossistema, avalia-se o nível de perda da biodiversidade.

O cálculo do coeficiente da perda da biodiversidade $\left(C_{P B}\right)$ é feito tomando-se a Equação (3) e substituindo so( $x$ ) pela função (8), em que: $x_{0}=$ área total do ecossistema (39.075 ha) e $x_{1}=$ área de vegetação suprimida (29.465 ha). Assim, tem-se que:

$$
C_{P B}=\frac{\int_{0}^{29465}\left[15,081 \cdot(39075-x)^{0,203}\right] d(x)}{\int_{0}^{39075}\left[15,081 \cdot(39075-x)^{0,203}\right] d(x)}
$$

Em que resulta em $C_{P B}=0,815$. Verifica-se, conforme a Tabela 1, que o resultado do coeficiente está no intervalo $0,8 \leq C_{P B} \leq 1$, o que implica que a perda da biodiversidade da Mata Atlântica em Viçosa do Ceará está em nível extremo.

O item a seguir apresenta os gráficos gerados a partir das equações apresentadas anteriormente. Para a elaboração dos gráficos e obtenção de valores numéricos para pontos das funções foi adotado o software gnuplot.

\section{RESULTADOS E DISCUSSÃO}

\subsection{Cenário estimado para perda da biodiversidade}

A Figura 4, gerada a partir das Equações (3), (4), (5) e (6), apresenta o decréscimo da quantidade de espécies de aves, mamíferos, répteis e o agrupamento dessas classes ao longo da devastação do ecossistema. À medida que o desmatamento avança e se aproxima da área total remanescente, o número de espécies decresce de forma mais acentuada, isto é, o desequilíbrio provocado pela destruição de habitats torna o ecossistema mais fragilizado e mais vulnerável à extinção, anulando a biodiversidade 
quando o desmatamento atinge a totalidade remanescente do ecossistema no momento atual (9.610 ha).

Figura 4 - Gráfico da estimativa de perda da biodiversidade no cenário atual

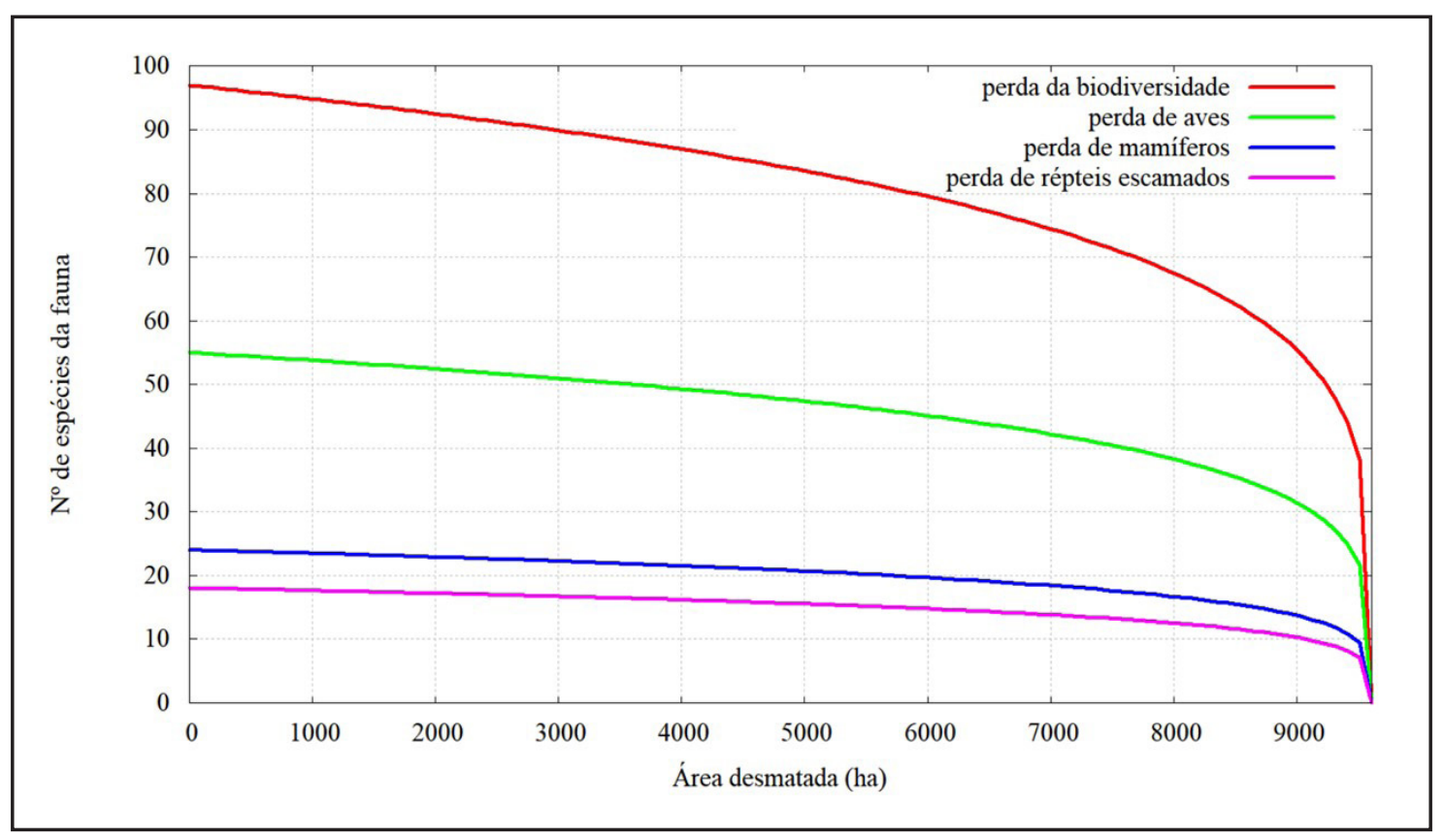

Fonte: Autores (2020)

De acordo com Scarano e Ceotto (2015), a perda de habitats naturais é provocada pela urbanização, industrialização e expansão agrícola. Para esses autores, um fato preocupante na manutenção da biodiversidade é que como a maioria dos fragmentos florestais da Mata Atlântica possuem tamanho reduzido, a sobrevivência de muitas espécies é comprometida (SCARANO; CEOTTO, 2015).

As pequenas populações de espécies são as mais suscetíveis ao processo de extinção local. Considerando a ocupação de habitats, simplesmente se expulsa a comunidade de animais para as regiões circunvizinhas (migração forçada), promovendo um aumento da densidade populacional e, consequentemente, reduzindo o alimento. A escassez de recursos provoca a competição tanto entre indivíduos de uma mesma população quanto entre espécies distintas. A competição entre populações provoca extinção, reduzindo a heterogeneidade do habitat. 
Entende-se que a classe de mamíferos é a mais vulnerável à perda da biodiversidade, pois comparando a relação das espécies locais no espaço temporal apresentada por Barros (1980), Ceará (1998) e IBAMA (1998), constata-se a extinção local das espécies Tamanduá-bandeira (Myrmecophaga tridactyla) e Tatu-canastra (Priodontes maximus), e baseado em Ibama(1998) e International Union for Conservation of Nature and Natural Resources (2020), verifica-se que a espécie Macaco Guariba (Alouatta ululata) está "em perigo" de extinção, e a espécie Gato-do-mato (Leopardus tigrinus) está "vulnerável" à extinção. As demais espécies de mamíferos, assim como as espécies de avifauna e répteis escamados estão na categoria "pouco preocupante" de risco de extinção (IBAMA, 1998; INTERNATIONAL UNION FOR CONSERVATION OF NATURE AND NATURAL RESOURCES, 2020).

\subsection{Cenário inicial de perda da biodiversidade}

Para Barros (1980), o processo de perda da biodiversidade em Viçosa do Ceará teve início com a chegada dos colonizadores, pois os silvícolas passaram a exterminar as espécies de animais de forma predatória, sem nenhuma cautela com a época de apreensão. Logo, esse processo de perda da biodiversidade poderia ter iniciado por volta do ano 1700, quando aconteceu a fundação da Aldeia da Ibiapaba (atual Viçosa do Ceará), intensificando o processo de intervenção antrópica no ambiente pelos colonizadores. A chegada dos europeus trouxe consigo práticas agrícolas que degradam o ambiente, levando a região à devastação de extensas áreas de floresta e extinção de espécies.

Como não se tem dados da cobertura vegetal em 1700, tomou-se como referência o ano de 1985, por ser o ano mais longínquo com dados da extensão da cobertura vegetal de Mata Atlântica em Viçosa do Ceará (FUNDAÇÃO SOS MATA ATLÂNTICA; INSTITUTO NACIONAL DE PESQUISAS ESPACIAIS, 2009). O município de Viçosa do Ceará possuía área original de Mata Atlântica equivalente a 39.075 ha, sendo que em 2014 identificou-se remanescente com apenas 9.610 ha (FUNDAÇÃO SOS MATA ATLÂNTICA; INSTITUTO NACIONAL DE PESQUISAS ESPACIAIS, 2015). Portanto, houve uma perda de 29.465 ha da cobertura original da Floresta Atlântica em um período correspondente 
a três décadas. A Figura 5, gerada a partir da Equação (8), apresenta dois momentos de perda da biodiversidade: a) o período de 1985 a 2014: representado no intervalo da abscissa que vai de 0 a 29.465 hectares, apontando uma moderada declividade referente à extinção local de espécies da fauna; b) a partir do ano de 2015: iniciando no valor da abscissa correspondente a 29.466 hectares, apresentando uma preocupante estimativa de perda de espécies.

Figura 5 - Gráfico da estimativa de perda da biodiversidade

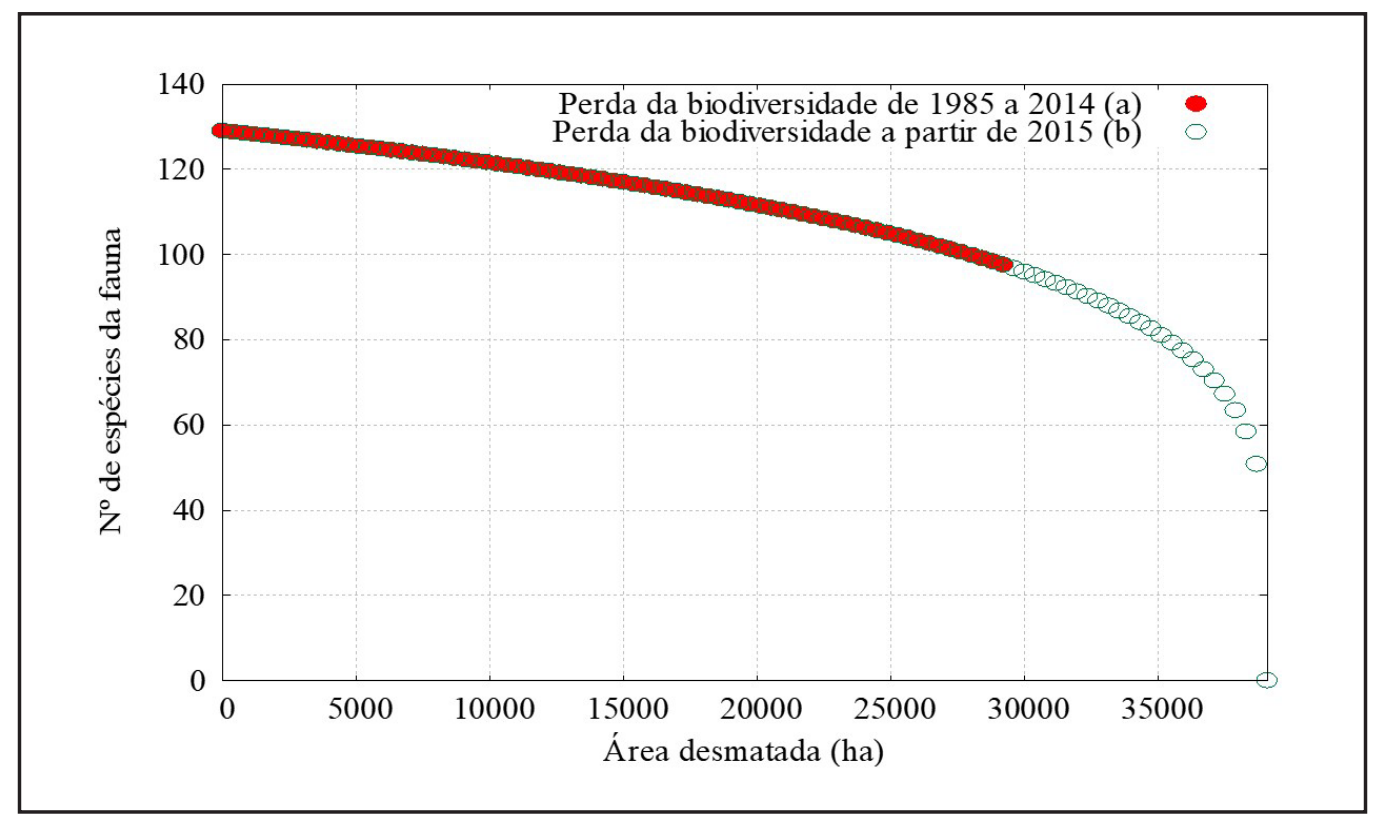

Fonte: Autores (2020)

De acordo com Rocha (2007), os fatores que vêm contribuindo para a extinção de espécies em Viçosa do Ceará são: a) caça predatória e comércio ilegal de animais silvestres; b) uso irracional e indiscriminado de agrotóxicos; c) desmatamentos e queimadas; d) expansão urbana; e) incorporação de novas terras ao sistema produtivo; f) presença de lixo doméstico e restos alimentares deixados pelos visitantes e turistas. A Figura 5 estima a perda de espécies em função da devastação de habitats, ou seja, em função dos itens c, d, e, mencionados por Rocha (2007). Logo, a ocupação de áreas naturais é a principal causa do decréscimo de riqueza de espécies (PURVES et al., 2005; HADDAD et al., 2015). 


\subsection{Taxa de variação média e taxa de variação da velocidade da perda da biodiversidade}

Nos gráficos apresentados nas Figuras 6 e 7, gerados a partir das Equações (10) e (12), respectivamente, observa-se um comportamento decrescente e taxas de variação média e de variação de velocidade quase nulas, mas em valores absolutos verifica-se aumento das taxas de variação média e de velocidade. Como as taxas de variação média e de velocidade têm o mesmo sinal, ou seja, ambas são negativas, o movimento é acelerado, pois o módulo dos valores está aumentando. O gráfico apresenta uma tendência de que quanto mais a área desmatada cresce, mais rápidas são a velocidade e a aceleração da perda da biodiversidade.

Figura 6 - Gráfico da taxa de variação média da perda da biodiversidade

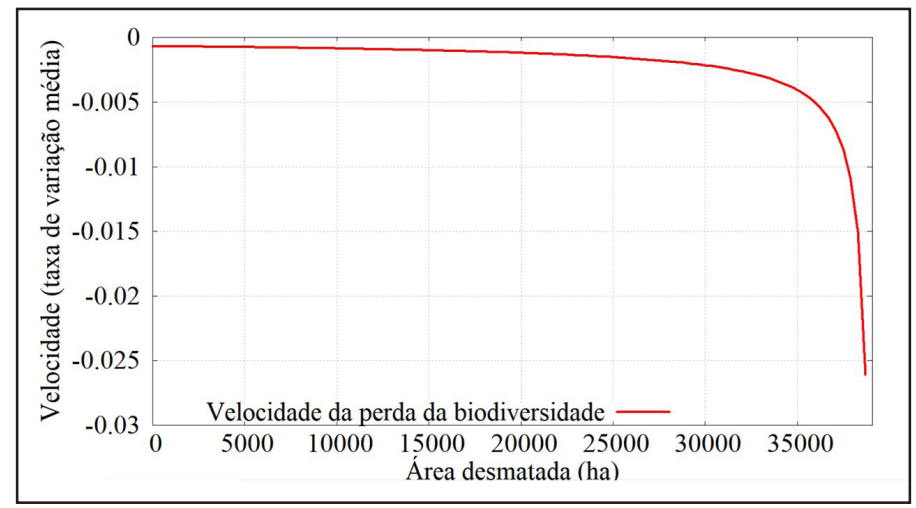

Fonte: Autores (2020)

Figura 7 - Gráfico da taxa de variação da velocidade da perda da biodiversidade

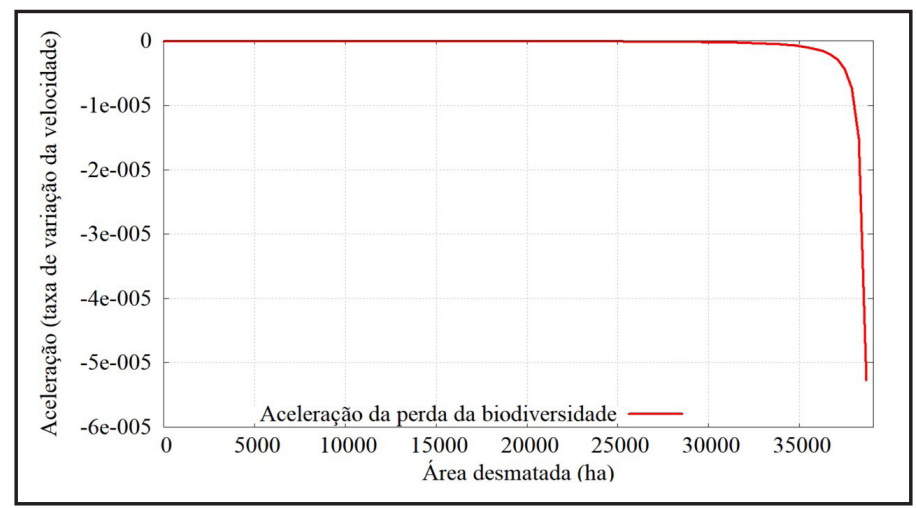

Fonte: Autores (2020) 
Sabe-se que é elevada a taxa de desmatamento das florestas tropicais para conversão da vegetação em plantações e pastagens. As taxas de extinção de espécies estão aumentando rapidamente (PURVES et al., 2005). O aumento da velocidade do desmatamento ocasiona a diminuição da área de forrageamento das espécies, provocando a migração de animais de grande porte para ecossistemas limítrofes, no município, como Caatinga e Carrasco. A eliminação da vegetação nativa também agrava o processo de isolamento do fragmento de mata úmida, tornando as espécies mais vulneráveis à extinção local.

Lévêque (1999) considera que certos ecossistemas, tais como as florestas tropicais e as espécies vegetais e animais que elas abrigam, desapareceram com uma velocidade acelerada. A diminuição das taxas de destruição de ecossistemas e extinção de espécies acontecerá quando a biodiversidade for valorizada como herança a ser transferida entre as gerações (PURVES et al., 2005). Segundo o Secretariado da Convenção sobre Diversidade Biológica (SCDB) (2010), as duas próximas décadas serão decisivas para determinar se as condições ambientais dos últimos 10.000 anos de civilização humana continuarão para além deste século.

\subsection{Curvatura e ponto crítico da perda da biodiversidade}

A Figura 8 mostra o comportamento da função da curvatura da perda da biodiversidade, generalizada para quaisquer ecossistemas - Equação (15). O ponto máximo da equação da curvatura $(k(x))$ corresponde ao momento crítico da perda, quando há uma inclinação mais acentuada no gráfico que apresenta o comportamento da perda da biodiversidade $(s o(x))$. A curvatura apresenta contínuo crescimento até a área devastada atingir 93\% do habitat, o ponto crítico da função, que representa o momento inicial de perda mais intensa.

O resultado do ponto crítico da Equação (17), apresentado pela Figura 8, coincide com o percentual aproximado de área desmatada no bioma de Mata Atlântica, ou seja, 93\%. Isso significa que o bioma atingiu o ponto crítico. O comportamento da 
perda até esse ponto é moderado, assim, apesar de o bioma Mata Atlântica possuir extensa área desmatada, ainda detém uma das maiores biodiversidades do planeta. No entanto, o desmatamento dos $7 \%$ que restam do ecossistema implica acelerada extinção de espécies.

Figura 8 - Curvatura referente à perda da biodiversidade

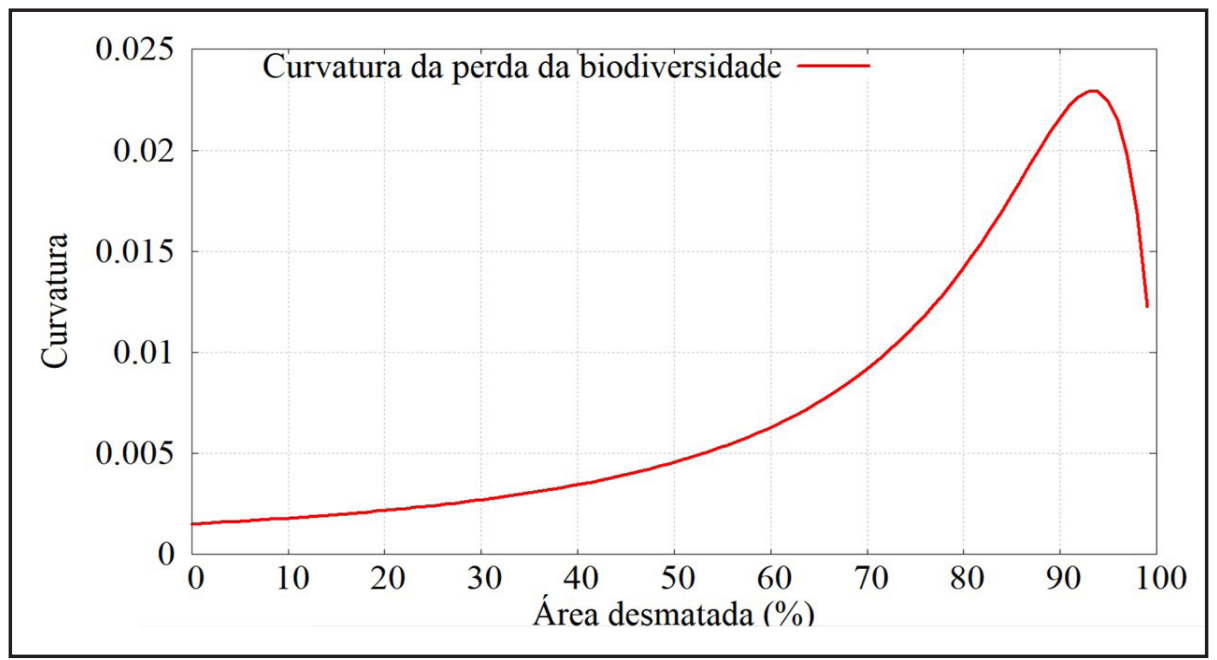

Fonte: Autores (2020)

De acordo com o Secretariado da Convenção sobre Diversidade Biológica (2010), quase metade (44\%) das ecorregiões terrestres tem menos de $10 \%$ de áreas protegidas, e muitas das regiões críticas para a biodiversidade continuam desprotegidas. O objetivo de proteger pelo menos $10 \%$ das regiões ecológicas do mundo para conservação de uma amostra significativa da biodiversidade está longe de ser cumprido.

\subsection{Coeficiente da perda da biodiversidade}

A comparação de cenários para o cálculo do coeficiente é apresentada na Figura 9, gerada pela função (8), que representa o cenário de perda do habitat a partir de 1985. A partir dessa comparação fica mais perceptível o nível de perda tanto da área com cobertura vegetal de Mata Atlântica quanto das espécies da fauna que habitam a localidade. 
O coeficiente da perda da biodiversidade, gerado a partir da Equação (19), apontou nível "extremo" de perda da biodiversidade no ecossistema de Mata Atlântica em Viçosa do Ceará, no período de 1985 a 2014. A Figura (9) mostra que o avanço de desmatamento a partir de 2015 tende a gerar intensidade da perda de espécies da fauna, o que torna a curva de perda da biodiversidade mais acentuada.

Figura 9 - Gráfico da perda da biodiversidade no ecossistema de Mata Atlântica, em Viçosa do Ceará

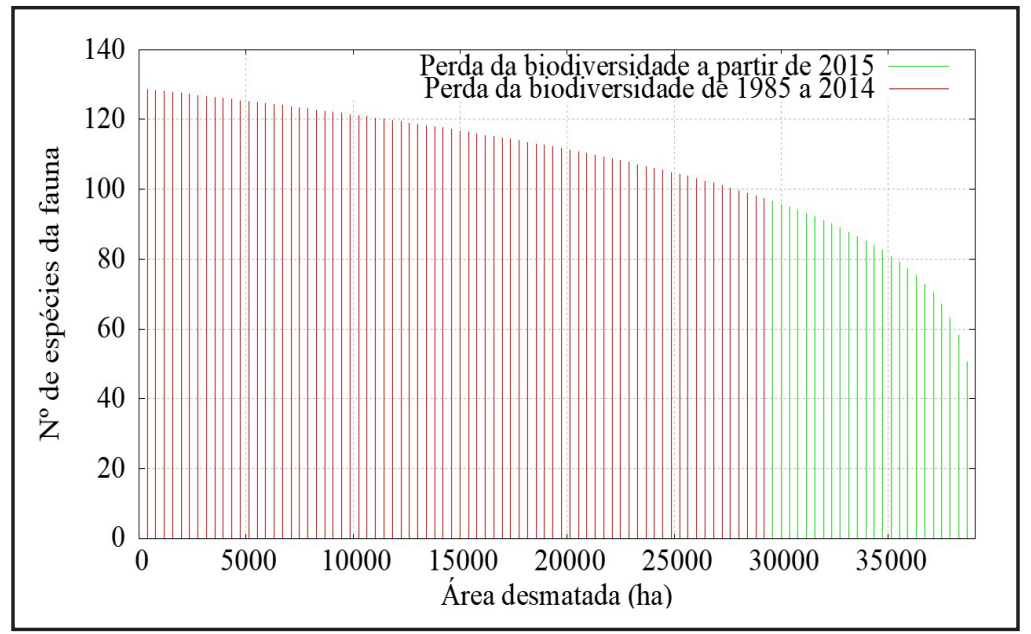

Fonte: Autores (2020)

Foi a acelerada redução dos ecossistemas naturais que motivou diversos estudos para investigar respostas da fauna à fragmentação da Mata Atlântica (UEZU, 2006). Uma pesquisa realizada no Estado do Amazonas para identificar o processo de extinção de espécies a partir da transformação de floresta em fragmentos isolados mostrou que os macacos foram as primeiras espécies a desaparecer dos fragmentos, pois necessitam de grandes extensões de áreas para habitar (PURVES et al., 2005). Das espécies de animais que habitam o município, a mais ameaçada é o Macaco Guariba (Alouatta ululata, Elliot, 1912), sendo a caça uma das principais causas de comprometimento da sobrevivência da espécie. Uma medida para resguardar a existência não apenas do Macaco Guariba (Alouatta ululata, Elliot, 1912), mas das demais espécies que habitam a localidade é através da criação de corredores ecológicos interligando matas isoladas. 


\section{CONCLUSÕES}

A partir do modelo apresentado foi possível verificar que na área em estudo as condições atuais de destruição da cobertura vegetal e extinção local de espécies da fauna, segundo o resultado do coeficiente de perda da biodiversidade, está em nível "extremo", sendo a categoria mais preocupante do coeficiente.

As taxas de variação média e de velocidade indicam que a curva da função da perda da biodiversidade tem comportamento de movimento acelerado. Assim, quanto maior é o crescimento da área desmatada, mais rápidas se tornam a velocidade e a aceleração da perda da biodiversidade no ecossistema.

O estudo da curvatura da função apontou como ponto crítico do processo de perda da biodiversidade, quando o ecossistema atinge 93\% da área desmatada, pois a partir desse ponto o comportamento da perda de riqueza de espécies acontece de forma mais acentuada.

Este estudo apresenta uma ferramenta de contribuição ao monitoramento do ecossistema e constitui um subsídio à sociedade, quanto à prestação de informação sobre o nível de perda de biodiversidade, a fim de que, com base nos resultados, seja definido o grau de urgência de implantação de programas de conservação e preservação dos ecossistemas naturais.

Como sugestão de trabalhos futuros propõe-se a aplicação do modelo a outros espaços detentores da Mata Atlântica. A disponibilidade de um maior número de estudos experimentais incrementaria uma base de dados de diferentes cenários, a fim de mitigar os impactos referentes às ações humanas e verificar a possibilidade de agregar novos indicadores ao modelo aqui apresentado.

\section{AGRADECIMENTOS}

Este artigo foi realizado com apoio da Coordenação de Aperfeiçoamento de Pessoal de Nível Superior - Brasil (CAPES) - Código de Financiamento 001. 


\section{REFERÊNCIAS}

ARROYO-RODRIGUES, V. et al. Multiple successional pathways in human-modified tropical landscapes: new insights from forest succession, forest fragmentation and landscape ecology research. Biological Reviews, Cambridge, v. 92, n. 1, p. 326-340, feb. 2017.

BARROS, L. T. História de Viçosa do Ceará. Fortaleza: Secretaria de Cultura e Desporto, 1980.

CARDINALE, B. J. Biodiversity loss and its impact on humanity. Nature,[s. I.],v. 486, p. 56-57, 2012.

CAVALCANTE, A. Jardins suspensos no sertão. Scientific American Brasil, São Paulo, v. 32, p. 60-67, jan. 2005.

CEARÁ. Superintendência Estadual do Meio Ambiente. Diagnóstico e macrozoneamento ambiental do Estado do Ceará. Fortaleza, 1998. v. 4.

CHAUDHARY, A.; MOOERS, A. O. Terrestrial Vertebrate Biodiversity Loss under Future Global Land Use Change Scenarios. Sustainability, [s. I.], v. 10, p. 1-20, aug. 2018.

CHISHOLM. R. A. et al. Species-area relationships and biodiversity loss in fragmented landscapes. Ecology Letters, Oxford, v. 21, p. 804-813, jun. 2018.

FERREIRA, I. J. M. et al. Landscape Landscape pattern changes over 25 years across a hotspot zone in southern Brazil, Southern Forests, [s. I.], v. 81, n. 2, p. 175-184, apr. 2019.

FUNDAÇÃO SOS MATA ATLÂNTICA; INSTITUTO NACIONAL DE PESQUISAS ESPACIAIS (Brasil). Atlas dos Municípios da Mata Atlântica - ano base 2014. [S. I.], 2015.Disponível em: https:// www.sosma.org.br/wp-content/uploads/2015/11/tabela-municipios-SOSMA_INPE_AtlasMunicipios_2014_rema.pdf. Acesso em: 22 dez. 2020.

FUNDAÇÃO SOS MATA ATLÂNTICA; INSTITUTO NACIONAL DE PESQUISAS ESPACIAIS (Brasil). Atlas dos Remanescentes Florestais da Mata Atlântica - período 2005-2008.São Paulo, 2009. $156 \mathrm{p}$.

HADDAD, N. M. et al. Habitat fragmentation and its lasting impact on Earth's ecosystems. Science Advances, [s. I.], v. 1, n. 2, e1500052, mar. 2015.

HOPPER, S. D.; SILVEIRA, F. A. O.; FIEDLER, P. L. Biodiversity hotspots and Ocbil theory. Plant and Soil, The Hague, v. 403, n. 1/2, p. 167-216, jun. 2016.

IBAMA. Plano de gestão e diagnóstico geoambiental e sócio-econômico da APA da Serra da Ibiapaba. Fortaleza: IESP; UECE, 1998. 100 p.

INTERNATIONAL UNION FOR CONSERVATION OF NATURE AND NATURAL RESOURCES. The IUCN Red List of Threatened Species. Version 2020-3. Cambridge, 2020. Disponível em: https://www.iucnredlist.org. Acesso em: 22 dez. 2020.

JURAS, I. A. G. M. Biodiversidade perdida: o desmatamento. In.: GANEM, R. S. Conservação da biodiversidade: Legislação e políticas públicas. Brasília: Edições Câmara, 2010. p. 415-432. 
LÉVÊQUE, C. A biodiversidade. Bauru: EDUSC, 1999. 245 p.

MACARTHUR, R. H.; WILSON, E. O. The theory of island biogeography.Pricenton: Princeton Landmarks in Biology, 2001. 203 p.

MACHADO, R. B. et al. Caracterização da fauna e flora do Cerrado. In: SIMPÓSIO NACIONAL SOBRE O CERRADO, 9.; SIMPÓSIO INTERNACIONAL SOBRE SAVANAS TROPICAIS, 2., 2008, Planaltina. Anais [...]. Planaltina: Embrapa Cerrados, 2008.

MYERS, N. et al. Biodiversity hostpots for conservation priorities. Nature, [s. I.], v. 403, p. 853$858,2000$.

PURVES, W. K. et al. Vida: a ciência da biologia. 6. ed. Porto Alegre: Artmed, 2005.

REZENDE, C. L. et al. From hotspot to hopespot: an opportunity for the Brazilian Atlantic Forest. Perspectives in Ecology and Conservation, [s. I.], v. 16, n. 4, p. 208-214, 2018.

ROCHA, F. C. Análise geoambiental do Município de Viçosa do Ceará. 2007. Dissertação (Mestrado em Desenvolvimento e Meio Ambiente) - Universidade Federal do Ceará, Fortaleza, 2007.

SAFAR, N. V. H.; MAGNAGO, L. F. S.; SCHAEFER, C. E. G. R. Resilience of lowland Atlantic forests in a highly fragmented landscape: insights on the temporal scale of landscape restoration. Forest Ecology and Management, Amsterdam, v. 470, 118183, aug. 2020.

SANTOS, A. J. Estimativas de riqueza em espécies. In: JUNIOR, L. C.; RUDRAN, R.; VALLADARESPADUA, C. (org.). Métodos de estudo em biologia da conservação e manejo da vida silvestre. Curitiba: UFPR; Fundação O Boticário de Proteção à Natureza, 2003. p. 19-41.

SANTOS, M. F. A. V. et al. Diversidade e densidade de espécies vegetais da Caatinga com diferentes graus de degradação no município de Floresta, Pernambuco, Brasil. Rodriguésia, Rio de Janeiro, v. 60, n. 2, p. 389-402, 2009.

SCARANO, F. R.; CEOTTO, P. Brazilian Atlantic forest: impact, vulnerability, and adaptation to climate change. Biodiversity and Conservation, London, v. 24, p. 2319-2331, 2015.

SECRETARIADO DA CONVENÇÃO SOBRE DIVERSIDADE BIOLÓGICA. Panorama da biodiversidade global 3. Brasília: Ministério do Meio Ambiente, Secretaria de Biodiversidade e Florestas, 2010. 94 p.

TABARELLI, M.; PINTO, S. R.; LEAL, I. R. Floresta atlântica nordestina: fragmentação, degeneração e perda da biodiversidade. Ciência Hoje, São Paulo, v. 44, n. 263, p. 36-41, 2009.

UEZU, A. Composição e estrutura da comunidade de aves na paisagem fragmentada do Pontal do Paranapanema. 2006. Tese (Doutorado em Ciências) - Universidade de São Paulo, São Paulo, 2006.

VEIGA, J. E.; EHLERS, E. Diversidade biológica e dinamismo econômico no meio rural. In: MAY, P. H.; LUSTOSA, M. C.; VINHA, V. (org.). Economia do meio ambiente: teoria e prática. Rio de Janeiro: Elsevier, 2003. p. 289-308. 


\section{Contribuição de Autoria}

\section{1 - Antonia Francivan Vieira Castelo Branco}

Graduada em Ciências - Hab. Matemática, Ma., Doutoranda

https://orcid.org/0000-0001-7461-4361・franvcb@hotmail.com

Contribuição: Conceituação, Curadoria de dados, Análise Formal, Software, Escrita primeira redação

\section{2 - Patrícia Verônica Pinheiro Sales Lima}

Engenheira Agrônoma, Dra.

https://orcid.org/0000-0002-6622-3640•pvpslima@gmail.com

Contribuição: Supervisão, Validação, Escrita - revisão e edição

\section{3 - Esdras Soares de Medeiros Filho}

Graduado em Computação, Dr.

https://orcid.org/0000-0003-2873-3583•esdras.med@gmail.com

Contribuição: Validação, Escrita - revisão e edição

\section{4 - Benedita Marta Gomes Costa}

Graduada em Ciências - Hab. Matemática, Dra.

https://orcid.org/0000-0002-2740-0560•martagcosta578@gmail.com

Contribuição: Supervisão, Escrita - revisão e edição

\section{5 - Tarcisio Praciano Pereira}

Graduado em Matemática, Dr.

https://orcid.org/0000-0003-0381-4133•pracianopereira@gmail.com

Contribuição: Software, Validação, Escrita - revisão e edição

\section{Como citar este artigo}

Branco, A. F. V. C.; Lima, P. V. P. S.; Medeiros Filho, E. S.; Costa, B. M. G.; Pereira, T. P. Avaliação da perda da biodiversidade na Mata Atlântica. Ciência Florestal, Santa Maria, v. 31, n. 4, p. 1885-1909, 2021. DOI 10.5902/1980509853310. Disponível em: https://doi.org/10.5902/1980509853310. 